vegetatión espontanea de la péninsula Hispano-Lusitanica é islas Baleares. Madrid. $8^{\circ} .32 \mathrm{~S}$.

Elfving F. Ueber die physiologische Fernwirkung einger Körper. Helsingfors. $4^{0} .18$ S. 2 phot. Taf.

Hansen A. Pflanzenphysiologie. Die Lebenserscheinungen und Lebensbedingungen der Pflanzen. $8^{\circ}$. 322 S. Illustr. Stultgart (O. Weisert). $6 \mathrm{Mk}$.

Keller R. Ueber Erscheinungen des normalen Haarverlustes an Vegetationsorganen der Gefässpflanzen. (Nova Acta Lcop. Carol. Akad. LV. Nr. 5). 44. 54 S. 3 Taf.

Kjellmann. Pflanzenbiologische Studien aus Russisch-Lappland. Beitrag zur Kenntniss der regionalen Gliederung und der polaren Waldgrenze. Holsingfors. $8^{\circ} .14$ Taf. 1 Karte. $-6 \mathrm{fl}$.

Kränzlin F. Xenia orchidacea. Beiträge zur Kenntniss der Orchideen von H. G. Reichenbach f. Fortgesetzt von. III. Bd. 4. Heft. Loipzig (F'. A. Brockhaus). 4. S. 65-76. 'Taf. 231--240.

Oliver J. W. Elementary botany. London (Blackie). $8^{0} .204$ S. 2 Sh.

Pierre L. Notes botaniques. Sapotacées. Paris (Klincksieck). 80. $36 \mathrm{~S}$.

Schimper A. F. W. Ueber Schutzmittel des Laubes gegen Transspiration, besonders in der Flora Javas. (Sitzungsber. d. k. preuss. Akad. d. Wissensch. XL.) Gr. 8". 18 S.

Steglich Dr. Zusammensetzung, Anwendung und Wirkung der Düngomittel. Dresden. $4^{\circ} .8 \mathrm{~S}$.

'T'schirch A. Physiologische Studien über die Samen, insbesondere über die Sangorgane derselben. Leiden (E. J. Brill). $8^{\circ} .40 \mathrm{~S}$. 6 Taf.

\title{
Erwiderung auf Dr. v. Wettstein's Besprechung meiner Flora von Niederösterreich.
}

Von Dr. Günther Ritter v. Beck.

College Dr. v. Wettstein hat den ersten Theil meiner Flora von Niederösterreich in dieser Zeitschrift einer Besprechung unterzogen, aus welcher zu entnehmen ist, dass ausser einigen flüchtig berührten Vorzügen in meinem Buche so ziemlich Alles nicht nach Wunsch des Referenten ausgeführt erscheint, dass im Besonderen: die Art der Standorts- und Verbreitungsangaben, die Anordnung des Stoffes, die Bestimmungstabellen, die Beschreibungen, die wissenschaftliche Nomenclatur, die Umgrenzung der Gattungen, die Nomenclatur der Arten die Unterscheidung der Formenkreise innerhalb der Arten 
die Unterscheidung und Benennung der Hybriden u. a. m. zu tadeln seien, ja man müsste, wenn man das Schlusswort des Kritikers liest, in welchem er , vor den Principien und Anschauungen, welche mir bei dor Abfassung meinos Workes massgebend waren, und überhaupt vor der Richtung meiner floristik warnt" wirklich glauben, dass mein Buch diesbezüglich geradezu verdammenswerth soi, gar keinen Fortschritt anfzuweisen hätte und nichts zu bieten hätte, was irgend einer besonderen Anerkennung würdig wäre, als die "reichste und beste Ausstattung".

Diese Ansichtsäusserungen meines Collegen erfordern meinerseits umsomehr eine Antwort, als Dr. v. Wettstein durchaus nicht in den Zweck meines Werkes, als "Handbuch zur Bestimmung sämmtlicher Gewächse Niederösterreichs" zu dienen, und in die diesbezüglich nothwendige Anordnung des Stoffes einzugehen beliebt, sondern bei der Beurtheilung meiner Flora einen Massstab anlegt, der insoferne für mein Werk unpassend erscheint, als selbst die besten Florenwerke und selbst Monographien bei einer derartigen Beurtheilung im Sinne des Kritiker's als ungenügend bozeichnet werden müssten.

Dass ein grösseres Werk nicht, allen $\Lambda$ nforderungen, namentlich woun sie nach persönlichen Ansichten oder nach gewissen Uebereinkommen gestellt werden, entsprechen kann, steht fest; ich muss abor gestehen, dass die wissenschaftlichen Anforderungen, wie sie Dr. Wettstein an eine Flora stellt, so weitgehende sind, dass ich vollends bezweifle, dass jemals eine Flora und sei es auch nur eine Localflora den Beifall Dr. Wettstein's finden dürfte, wenn dieselbe nach denselben Gesichtspunkten beurthoilt würde, wie mein Handbuch. Ich glaube mit Recht aussprechen zu können dass solche wünschenswerthe Ziele wie z. B. die richtige endgiltige Umgrenzung der Gattungen, die Unterscheidung der Formenkreise innerhalb einer Art nach ihrer Entwickelung u. a. eber ausgesprochen als erreicht sind, Niemandem aber die Nichterreichung derselben zum Vorwurfe gemacht werden kann, umsomehr, als eine diesbezüglich entsprechende Flora weder von Dr. Wettstein bisher geliefert warde noch überhaupt existirt.

Ich will nun auf die Vorwürfe näher eingehen und hiebei deren Begründung beleuchten.

Dr. Wettstein behauptet vorerst, dass mir die vorliegenden Verbreitungsangaben ,ein reiches Material abgegeben hätten, um insbesondere gewisse Eigenthünlichkeiten der horizontalen und verticalen Verbreitung, die Ablängigkeit von Klima und Boden zu fixiren, dass meine allzu allgemeinen Angaben jedoch dies nicht vermögen, und dass ich überhaupt die Verbreitungsangabeu zu leicht abgethan habe". Dies ist nicht zutreffend. Mir lagen alle Verbreitungsangahen für eine Art vor, diese prïfte ich an der Hand einer pflanzengeographischen, geologischen und klimatologischen 
Karte und gelangte anf diese Weise zur Erkenutniss, dass sich für gewisse, häufiger, doch zerstreut vorkommende Pflanzen ob ihres sporadischen Vorkommens auf verschiedenen Unterlagen, in verschiedenen Pflanzenregionen und Florengebieten keine Gesetzmässigkeit des Vorkommens in unserem doch relativ sehr gut bekannten Lande eruiren lässt. Ich bin mir auch bewusst, keine für die Pflanzengeographie Mitteleuropas wichtige Thatsache übergangen zu haben.

Uebrigens ersieht man aus jeder meiner auch allgemein gehaltenen Angabe 1. die Pflanzenformation (Wiese, Vorhölzer, Wälder etc.) in welcher sich eine Pflanze vorfindet; 2. die vertivale Verbreitung, bis zu welcher Region ${ }^{1}$ ) sie verbreitet ist (vielfach sind noch Angaben über die obere Höhengrenze eingeschaltet); 3. die horizontale Verbreitung d.h. wenigstens die Begrenzung des Areales, Vegetationslinien im Allgemeinen; 4. das Florenge bie z. B. pamnonische Flora (es deckt sich aber oft mit den Regionen; für die mitteleuropäische (baltische) Flora entfiel die Nennung); 5. die Abhängigkeit der Pflanze von der geognostischen Unterlage, wo sie ermittelt werden konnte; 6. die Art des Auftretens (häufig, selten in Beständen, etc.); 7. die Blüthezeit.") Es scheint mir daher Dr. Wettstein's Ansicht, dass jch "die Sache za leicht abgethan" habe, nicht gerechtfertigt.

Dass ich die Finder nicht nannte, gebe ich zu. $\left.{ }^{3}\right)$ Dass ich dies in einem Bestimmungsbuche nicht hätte thun dürfen, bevor ich nicht die Belegexemplare gesehen hätte, bezweifle ich. Selbst Noilreich nennt sie nur in Ausnahmsfällen, in Grenier's und Godron's Flora oder Koch's Synopsis u. a. fehlen sie. Meiner Ansicht nach muss man Angaben von Pflanzen, deren Vorkommen möglich ist, so lange für wahr halten, bis man sich nicht an der Hand von

$\left.{ }^{1}\right)$ Diese sind schon in meiner Flora von Hernstein pflanzengeographisch und klimatologisch festgestellt.

2) Selbst manche Standortsangaben in den Specialarbeiten Dr. Wetts tein's geben nicht über alle diese Verhältnisse Aufschluss. So erfährt man bei den Sesterien (Abh. zool.-bot. Ges. 1888) z. B. nichts über deren verticale Verbreitung, über Blüthezeit.

3) Dr. Wettstein meint, ich hätte hiefür Platz gewonnen, wenn jch die nachgewiesenermassen im Kronlande noch nicht gefundene Nigritella fragrans oder die nicht mehr vorkommenden Delphinium elatum, Silene saxifraga u. a. weggelassen hätte. Nun ist der Nachweis, dass Nigritella fragrans noch nicht gefunden worden sei, noch nirgends erbracht worden, denn Kerner (in Abh. zool.-bot. Ges. XV, S. 219) glaubt das Vorkommen nur in Zweifel zu ziehen, und Wettstein (in Ber. deutsch. bot. Ges. 1889, S. 314-315) hat die betreffenden Originalexemplare Dolliner's von der Raxalpe ebensowenig wie Kerner gesehen, kann daher nicht behaupten, dass eine Hybride, deren Stammeltern bei uns häufig sind, nachgewiesenermassen bei uns nicht vorkommt. Delphinium elatum ist schon von Neilreich aufgenommen worden, übrigens durch Kleindruck in meiner Flora als fremd bezeichnet, und Silene saaifraga konnte $i c h$ als eine im angrenzenden Gebiete vorkommende Pfanze ebensowenig weglassen wie die Flora des Nensiedlersees. Ich bedauere daher, den Rathschlag Dr. Wettstein's nicht befolgen zu können, indem die. Weglassung dieser Pflanzen wirklich begründete Forwürfe nach sich ziehen würde.

0esterr. botan. Zeitschrift. 3. Heft 1891. 
Originalexemplaren von der Unrichtigkeit derselben überzengen kann.

Allgemeine Bemerkungen Wettstein's, wie, dass die eigenthümliche Anordnung des Stoffes ilm nicht nachahmenswerth sei, dass die Bestimmungstabellen und Beschreibungen viel zu schwer verständlich seien u. a., brauche ich, als nicht weiter bogründot, nicht zu widerlegen. Dr. Wettstein behauptet aber, „dass die von mir gegebene Charakteristik der Gymnospermen für einen Botaniker nicht nöthig gewesen wäre, und dass der Anfänger derselbon am Beginne eines Handbuches zur Bestimmung wie einer räthselhaften und ihm unlesbaren Inschrift gegenübersteht". Den ersten Theil dieser Aeusserung kann ich nicht recht bogreifen, denn meines Dafürhaltens ist eine sorgfältige Gliederung des Stoffes ohne eine Charakteristik der Gruppen überhaupt nicht möglich, und wenn ich dieselbe ansführlicher hielt und namentlich gebräuchlichere "Termini technici und Erläuterungen in Parenthese einfïgte, kann das ja nur ein Vortheil sein.

Bezüglich des zweiten Theiles der vorigen Aeusserung will ich erwäbnon, dass ich auch bei einem Anfänger bescheidene Vorkenntnisse voraussetze, denn Pflanzenbestimmen ist gewiss keine Kinderspielerei, und dass ich im Vorworte zu meinem Buche erwähnte, dass dem allgemeinen Theile des Werkes ein kurzgefasster Abschnitt über Organographie zum besseren Verständnisse des Textes beigegeben werden wird.

Bezüglich der Gattungsumgrenzung spricht Dr. Wettstein die schwersten Vorwürfe gegen meine Flora aus, indem er sagt, jch habe dieselbe , vach Zweckmässigkeitsgründen und nicht nach wissenschaftlichen Grundsätzen, nicht auf Grund sämmtlicher in Betracht zu ziehender Formen, sondern nur mit Berücksichtigung der Formen des Florengebietes vorgenommen und so eine ganze Reihe unhalbarer Gattungen aufgestellt oder restituirt, die nichts weiter als eine Verwirrung der Nomenolatur bewirken werden."

Es wird mir schwer, kuxz darauf zu antworten. Ich kann nur darauf hinweisen, dass Koch, Grenier, Godron, Colakovsky und andere Forscher, denen wir unsere besten Florenwerke verdanken, anch nicht anders vorgegangen sind als ich, trotzdem aber mit ihrer Gattungsumgrenzung nicht übereinstimmen, dass ich bei der Behandlung der Gattungen stets unsere besten Werke, wie Bentham und Hooker's Genera, die natürlichen Pflanzenfamilien u. a. zu Ratho zog and nur dort Trennungen vornahm, wo es mir nach der bisher in don einzelnen Familien festgehaltenen Gattungsumgrenzung zweckdienlich oder geboten erschien. Auf diese Weise habe ich manche Soction wieder als Gattung restituirt, weil mix die Charakterunterschiode zwischen den Sectionen viel grösser und gehaltvoller erschienen, als die generellen Unterschiede dex benachbarten Gattungen und weiters, weil moine Anschauung dahin geht, dass der in den einzehnon Familien verschiolone Gattungsbegriff wenigstens in dersolben Familie bestmöglichst nach gleichen Grundsätzen festgostellt werdon soll. Wenn Dr. Wettstein mir vorwirft, dass ich ,im Gegensatze 
zu den äblichen Ansclanungen bei einigen Famjlien kleine Gattungen liebe, bei anderen wieder ein Freund grosser Gattungen bin", so möchte ich ihn fragen, ob es überhaupt möglich sei, die schwach begrenzten Gattungen der Compositae, Gramineae und anderer Familien etwa mit den uralten Gattungstypen der Cupuliferae n. a. wesentlich gleich zu machen? Das bringt doch wohl auch die bestens geartete Systematik nicht zu Stande! Ich begreife aber sehr wohl, warum Dr. Wettstein gegen meine Gattungsumgrenzung eifert. E'rüher ein Freund kleiner Gattungen, ${ }^{1}$ ) ist er nun in seinen neneren Arbeiten Liebhaber von Gattungen mit grösstem Umfange geworden. ${ }^{2}$ ) Ich glaube kaum, dass solche Gattungen, wie sie Wettstein in der Familie der Orchidaceae liebt, und deren Umfang wir nur vorläufig ahnen können, $\left.{ }^{3}\right)$ Nachahmer finden dürften.

Zum Beweise des über meinen Gattungsbegriff Ausgesagten hat Wettstein eine Parallele zwischen meiner Charakteristik der beiden Gattungen Leucojum und Erinosma gegeben. Ich muss es jedoch als irrthümlich erklären, dass ich Masse in die Gattungsdiagnose anfoenommen habe, ${ }^{4}$ ) und weiter's bemerken, dass die Wiedergabe der Diagnosen insoferne unvollständig ist, als Wettstein ein Merkmal des Samens wegliess. Bezüglich der Placentation beider Pflanzen, Leucojum aestivum und Erinosma vernum, gebe ich zu, dass meine Zeichnungen diese Verhältnisse etwas übertrieben darstellen, nach erneuerten Untersuchungen behaupte ich jedoch noch immer:

1. dass die Gestalt der Samenknospen und deren Einfügung hei beiden Gattungen verschieden ist.

Bei der Gattung Leucojum sind die Samenknospen allseitswendig (d. h. die oberste anfsteigend, die unterste absteigend, die mittleren mehr minder wagrecht) und an der Chalaza abgerundet. Hingegen bei der Gattung Erinosma sind die Samenknospen sämmtlich mehr minder anfsteigend und an der Chalaza zugespitzt und Wettstein's Zeichnung Fig. 4 ist nicht genau.

2. Die Unterschiede in der Samenbildung sind sehr auffällige.

Ich kann nicht begreifen, wie Wettstein über die Samon kurzweg zu urtheilen vermag, wenn er nach eigener Aussage die Samen der einen Art nicht gesehen hat, während ihm von der anderen Art

1) Dr. Wettstein hat deren mehrere von kleinstem Unfange zum Theile mit Dr. O. Stapf aufgestellt.

$\left.{ }^{2}\right)$ Dass Dr. Wettstein bei Begrenzung derselben nicht immer wissenschaftlich zu Werke geht, ersieht man aus der Begründung, mit welcher er die Gattung Ionorchis (= Limodorum Tourn.) zu Epipactis zieht, indem er hiefür nur anführt: "Niemand der Epipactis cucullata sah, kann daran zweifelı, dass Limodorum unbedingt mit dieser in eine Gattung gehört".

$\left.{ }^{3}\right)$ Wenn Wettstein z. B. Nigritella zu Gymnadenia zieht, so müssen nach gleichen Grundsätzen, Orchis zu Gymnadenia und dann Anacamptis, Chamaeorchis und eine Reihe anderer Gattungen dazu gezogen werden.

$\left.{ }^{4}\right)$ Wettstein hat dies zwar in einer Anmerkung auch selbst erklärt. 
offenbar nur schlechtes Material vorlag. Herbert hat hauptsächlich wegen der Merkmale der Samen seine Gattung Erinosma aufgestellt, und meiner Ansicht nach mit Recht, denn Leucojum aestivum hat fast kugelige, auffällig grössere, $5-6 \mathrm{~mm}$ messende, rundum abgerundete, anhangslose, glänzend schwarzo Samen und ein hornartiges Nährgowebe, in welchem der grosse fast ${ }^{3} / 4$ so lange Keimling liegt. Die Samenschale besteht im unteren Theile des Samens aus 4 Laigen, von denen die 2 äusseren aus kohlschwarzen 'Tafelzelleu bestehen, sich von der inneren Hälfte der Testa trennen, und den Sumen als brüchige, glänzend schwarze Hant rundum lose umgeben. ${ }^{1}$ )

Hingegen hat Erinosma vernum als einzige Art dieser Gattung ellipsoidische, ohne Auhängsel $3-3.5 \mathrm{~mm}$ lange Samen, welche stets ein (nicht abfallendes!) kegelförmiges, $1 \cdot 5-2 \mathrm{~mm}$ langes Chalazaanhängsel tragen. Die Samenschale besteht nur aus einer Lage wenig verdickter 'Tafelzellen, welche dem Samenkerne fost aufliegen und dem Samen ein mattes, braunes, etwas körniges Aussehen verleihen. Das Nährgewebe ist gebräunt, fast holzartig und der Keimling sehr klein, kaum $1 / 3$ so lang als dasselbe.

Hs ist möglich, dass diese hier nochmals vorgebrachten guten Differentialmerkmale in den Angen Dr. v. Wettstein's vielleicht noch immer zu geringfügig erscheinen, um die Trennung der beiden Gattungen zu rechtfertigen; sie sind aber meines Dafürhaltens noeh immer besser als die Unterschiede zwischen Cirsium und Carduus, Hieracium und Crepis, Ornithogalum und Gagea und vieler anderer gebräuchlicher Gattungen. Wenn aber Wettstein ausdrücklich hervorhebt, dass in den genannten Pflanzen nur zwei gut unterscheidbare Arten, nie und nimmer aber zwei Gattungen vorliogen", so kann dazu bemerkt werden, dass, abgesehen von Herbert, der die Gattung Erinosma begründete, und von dem kritischon Kunth (Enum. V), der sie beibehielt, Bentham und Hooker (Gen. III, S. 720) und Pax (Nat. Pflanzenfam. II, 5, S. 105) die beiden Arten in zwei Sectionen Baker (Amaryll. S. 19) aber in zwoi Untergattungen untergebracht haben, die ich wie die 3. Section als selbstständige Gattungen auffasse.

Ich muss nun auch die leidige Nomenclatur der Arton berühren, die Wettstein selbst als nebensächlich bezeichnet, aber trotzdem des Weiten ausführt, um beweisen zu wollen, dass ich mit meinem Grundsatze, jenen Speciesnamen einer Pflanze anzunehmen, wolcher ihr zuerst in der richtigen Gattung gegeben worden ist, vollends Unrecht habe, dass hiedurch die Nomenclatur der Arton nie eine Stabilität erreichen wird ete.

Dagegen meint Wettstein, dass das einzige richtige Prioritätsprincip in der rücksichtslosen Anwendung der ältesten Speciesnamen liege, gleichgiltig in welcher Gattung immer derselbe gegeben

1) Die zwoite Art dieser Gattung $L$. Hernandezianum Camb, ist wohl nur eine schmalbläiterige Form von $L$, aestivum. 
worden sei, ${ }^{1}$ ) nur so würde weiteren Aendermngen in der Nomenclatur vorgebeugt, meine dagegen vorgebrachten Motive ${ }^{2}$ ) seien durchaus nicht massgebend u. s. w.

Jeder Botaniker weiss, dass bezüglich der Nomenclatur der Arten vielerlei Ansichten herrschen, die ihe pro und contra haben, und dass es bisher trotz aller Congresse und Torschläge nicht möglich war, eine Finigkeit zu erzielen. Man liess daher allen diesen Richtungen freien Lauf, wemn sie nur den binären Grundsätzen entsprachen. Es ist ja auch ganz nebensächlich ob eine Pflanze gerade den ältesten oder einen anderen Namen trägt, wenn sie nur durch die Benennung klar bezoichnet ist. Sache des Uebereinkommens ist es, diesen oder jenen Namen zu wählon. Richtig sind allo. Unlogisch dürfte os jodoch sein, wenn Wettstein meint, dass ich durch den Wiedergebranch des (ältesten) Speciesnamen "abortius" bei der Umtanfung der Gattung Limodorum in Jonorchis selbst dio Löslichkeit eines binären Pflanzennamens bewiesen hätte. Richtig wäro das nur dann, wenn jeder Speciesnamen nur einmal im Pfanzenreicho vorkommen würde! Oder sollen die Speciesnamon albus, niger, acer u. s. w., deren Wiedergebrauch allgemein ist, für sich allein etwas mehr bedenten als irgend eine Eigenschaft? Doch erst in ihrer Verbindung mit einom Gattungsnamen erlangen sio auf Grund von gegebenen Merkmalen systematischen Werth ind verlicren demnach denselben auch wieder bei ihrer Trennung.

Die mir zum Vorwurfe gemachte Differenz in der Nomenclatur der Arten ist übrigens gar nicht so bedentend.

Ich benütze ebenfalls dem Gebrauche gemäss den ältesten Speciesnamen bei der Uebersetzung einer Pflanze in ein neues Genus, aber ich anerkenne nicht das "Müssen“, das Dr. Wettstein als Gesetz hinstellt. Namentlich sehe ich nicht ein, warum ein nach allen Regeln richtig gebrauchter Speciesnamen in der richtigen Gattung umgeworfen werden soll, weil man nachgewiesen hat, dass dio Pflanze bereits in einer ganz entfernten Gattung einen anderen Artnamen besass. Der älteste Speciesnamen in der riehtigen Gattung wird stets unumstösslich bleiben, so lange wir nicht den Art- oder den Gattungsbegriff ändern, was oben bei dieser und bei jener Nomenclatur immer vorkommen wird. Beim bedingungslosen Gebrauche des überhaupt ältesten Speciesnamen ist man aber niemals sicher, ob nicht doch noch ein älterer in einer fremden Gattung gefunden werden dürfte, wofür ich mehrere Beispiele anführen könnte.

Anderntheils erachte ich die Anwendung des überhaupt ältesten Speciesnamens mit so vielen Ausnahmen behaftet und mit so vielen

1) Wenn dies wirklich der Fall wäre, warum hat es gerade ein Vertreter dieses Grundsatzes; Prof. A scherson, wieder aufgegeben? Warum nehmen es viele englische, französische, deutsche Autoren a. a. nicht an?

$\left.{ }^{2}\right)$ Monographie der Gattung Orobandhe S. $61 \mathrm{ff}$. 
Widorsprüchen bogleitet, ${ }^{1}$ ) dass ich schon aus diesen Gründen mich mit dieser Nomenclatur nicht befreunden kann.

In Bezug auf die Unterscheidung engerer Formenkreise iunerhalb einer $\Lambda$ rt, wirft mir Wettstein vor, dass jch durch kritiklose Nebeneinanderstellung derselben in manchen Fällen trotz vorliegender Arbeiten wieder völlige Unklarheit geschaffen habo, und dass ich oft mehr behaupte, als sich beweisen lässt.

Beweise hiefür soll Wottstein's Arbeit über Seslerir coerulea lieforn, der ich nicht gefolgt biu. Abgesehen ron den bei dieser Gelegenheit anfoestellten Behauptungen des Kritikers, welche so scharf sind, dass ich sie als persönliche Beleidigungen ansehen muss, die aber trotzdem nicht nachwoishar sind. weil joh eben an meinem von Wettstein nicht geprïften Materiale und nach meinen Beobachtungen zu anderen Resultaten celangte, wird es Niemand für unwahrscheinlich halten, dass zwischen den beiden jüngst von Wettstein wieder als Arten getrennten Seslerien"2) die nur relativ in don Vegetatiousorganen gering differenzirt sinı, Uebergangsformen möglich sind. Ich behanpte aber nach ermenerter Revision meines Herbares auch heute noch, dass Uebergangsformen zwischen allen von mir in meiner Florabehandelton Formen der Sesleria coerulea existiren.

Den Tadel den Wettstein in der Anwendung des Epithetons typicus findet, mit welchem ich die zuerst binär benannte Form einer Pflan\%e der Einfachheit halber bezeichnete, halte ich für belanglos; ich muss mich jedoch dagegen wenden, wenn der Kritiker moine Unterscheidung und Benennung der Hybriden als solche von Individuen ansieht und mir vorhält, dass zwischen zwei A rten nach der Beobachtung nur zwei Formenkreise von Hybriden unterschieden werden können. Nach meiner und anderer

1) Als Beispiel hiefür führe ich nur einige Benennungen aus Wett. stein's soeben erschienenem Lehrbuche an, in welchem doch vor Allem a uf' richtige Benennungen Rücksicht zu nehmen war. Die Weisstanne heisst darin Abies pectinata. Warum nicht Abies Picea, da dieselbe doch bei I in né Pinus Picea heisst? Warum wird der aus dem Jahre $180 \%$ stammende Speciesname gewäblt, wo doch Miller diese Pflanze schon 1768 als Abies alba bezichnete? Gattungsnamen werden doch wiederholt von Wettstein als Artennamen gebraucht, wie z. B. bei Quevcus Ilex, Acorus calamus, Dianthus caryophyllus! - Die "Roth"-Fichte heisst Abies excelsa. Abgesehen davon, dass doch Eichler (nat. Pflanzenfam.) die Gattung Picea scharf charakterisirte, müsste sie doch Abies abies heissen. Die Zoologen dieser Nomenclaturrichtung perhorresciren durchaus nicht die Wiederverwendung des gleichlautenden Speciesnamens! - Die Brunnkresse heisst Nasturtium offleinale. Abgesehen davon, dass der Gattungsname $N_{\text {asturtium }}$ ganz unhaltbar ist, müsste doch nach Wettstein's Grundsätzen die Pflanze $N$. fontanum heissen (wie es Ascherson nach gleichen Grundsätzen in seiner Flora von Brandenburg gethan), wenn man schon Linne's Speciesnamen "Nasturtium aquaticum" nicht anwendet, und den nächst älteren zur Verwendung bringt. Unsere Linden werden Tilia grandifolia und $T$. parvifolia genannt, wo doch viel altere Speciesnamen existiren, u. s. w.

2) Nach Celakovskj (Resultate 1888) sind es nur Rassen. 
Anschanung sind zwischen zwei Arten unendlich viele Hybride möglich und in gewissen Fällen auch nachgewiesen wio \%. B. zwischen Cirsium palustre und C. oleraceum, Salix alha und S. fragilis u. a. In solchen Fällen wird es unmöglich, nur zwei Formenkreise zu unterscheiden, man wird sich begnügen müssen, einzelne auffallige Hybride aus der Unzahl hervorzuheben, wenn man in eine nähere Unterscheidung der einzelnen Hybriden eingehen will, wenn nicht, dann ist zur Bezeichnumg dersolben nur ein Name richtig, und das ist der aus dem Speciesnamen der beiden Stammeltern zusammengesetzte. Korner, dem wir vielfach dio Klärung der Bonennung der Hybriden verdanken, hat die einzehen Hybriden zwischen zwei gleichen Stammarten stets nach Art der Arten verschieden und ofters mehrere benannt; nach meiner Ansicht mit Recht, so lange sie sich interscheiden lassen, denn Hybride sind Znfallsbildungen, die bald mehr bald weniger an Merkmalen ihrer Stammeltern aufweisen, aber gewiss nicht, wie Wettstein moint für sich melr variiren als die Arten. Da nun oftmals nur ein einziges Exemplar einer Hybride bekannt ist, muss die Beschreibung unbedingt eine Individuenbeschreibung soin. Wenn aber eine andere Fybride aus muthmasslich denselben Stammeltern ganz andere Merkmale zeigt als dio bisher bekanten Hybriden, ja sogar Morkmale aufweist, die beiden Stammeltorn fehlon, so ist es gewiss anch nach dem Gebrauche nicht gerechtfertigt, sio mit demsolben Namen zu bezeichnen; ausgenommen, man benennt sio mit jenem der muthmasslichen Stammeltern. Ioh habe boi der Benennung der Hybriden beiden Richtungen entsprochen und glanbe hiermit dem allgemeinen Gebrauche gemäss vorgegangesl zu sein.

Wenn ich hiermit die Widerlegung und Klärung der hauptsächlichsten Vorwürfe des Kritikers beschliesse, so geschiehti es aus dem Grunde, weil ich die zahlreichen anderen Bemerkungen Dr. v. Wettstein's als zu vebensächlich ansehe, und weil ich fürchte, den Raum einer sachlichen Erwiderung ohnehin schon überschritten zu haben.

Ich muss aber am Schlusse nochmals betonen, was ich schon Eingangs hervorhob, dass Dr. v. Wettstein oinestheils an mein Handbuch Anforderungen stellte, die noch ron keiner Flora erreicht worden sind, anderentheils bei seinen Ausführungen sich allzusehr auf persönliche Anschauungen stützte, die keineswegs noch genügend bekräftigt oder allgemein angenommen sind. Aus diesem Grunde scheinen mir Aeusserungen eines so scharfen Tadels wenig gerechtfertigt, namentlich aber muss ich zur Wahrung meiner wissenschaftlichen Ehre und mit Rücksicht auf das in meiner Flora Geleistete betonen, dass in keinem Falle eine zwingende Pflicht vorlag, vor der in meinem Buche vertretenen Richtung der Floristik zu warnen, umsomehr, als Dr. v. Wettstein mit keinem Worte das berïhrte, worin ich moinen Stolz finde, nämlich die Originalität des ganzen Werkes.

Wien, am 9. Februar 1891. 